\title{
Prevalence and associated factors of depression in patients with psoriasis at psoriasis clinic in a university hospital in
}

Thailand

Kamonporn Wannarit ${ }^{\star}$, Chanisada Wongpraparut ${ }^{\star \star}$, Leena Chularojanamontri ${ }^{\star \star}$ Narumol Silpa-archa**, Nutchanart Tayeh*, Punyisa Prachgosin*, Woraphat Ratta-apha*

* Department of Psychiatry, Faculty of medicine, Siriraj Hospital, Mahidol University.

** Department of Dermatology, Faculty of medicine, Siriraj Hospital, Mahidol University.

\section{Background}

Patients with psoriasis not only suffer from several physically burdensome symptoms, but also from depression and stress. In addition, psoriasis may affect their quality of life as well.

\section{Objectives}

To investigate the prevalence and associated factors of depression in patients with psoriasis.

\section{Materials and methods}

Patients with psoriasis were invited to complete the Thai versions of Patient Health Questionnaire (PHQ-9), Perceived Stress Scale-10, Alcohol Use Disorders Identification Test, Fagerström Test for Nicotine Dependence, Psoriasis Disability Index (PDI), Psoriasis Area and Severity Index and Simplified Psoriasis Index (SPI). T-test and chisquare test were used to examine associated factors of depression. Pearson's correlation was used to investigate relationship between severity of depression and other mental health problems.

Table 1: Comparison of psoriasis impact on participants who had depression and did not have depression using t-test $(\mathrm{n}=150)$

\begin{tabular}{|c|c|c|c|}
\hline Variable & $\begin{array}{c}\text { Participants who } \\
\text { had depression } \\
(\mathrm{N}=32)\end{array}$ & $\begin{array}{l}\text { Participants who did } \\
\text { not have depression } \\
\qquad(\mathrm{N}=118)\end{array}$ & $P$-value \\
\hline & Mean \pm SD & Mean \pm SD & \\
\hline aT-PSS-10 & $17.94 \pm 4.50$ & $15.08 \pm 5.40$ & $0.007 *$ \\
\hline bPASI & $11.080 \pm 9.01$ & $8.79 \pm 8.35$ & 0.190 \\
\hline cproSPI Severity & $12.69 \pm 7.84$ & $10.75 \pm 9.93$ & 0.150 \\
\hline cproSPI Psychosocial & $5.58 \pm 3.07$ & $3.76 \pm 2.75$ & $0.002 *$ \\
\hline cproSPI Intervention & $3.29 \pm 2.08$ & $2.91 \pm 2.02$ & 0.363 \\
\hline${ }^{\mathrm{d}}$ saSPI Severity & $12.24 \pm 10.89$ & $6.80 \pm 7.35$ & $0.001^{*}$ \\
\hline${ }^{\mathrm{d}}$ saSPI Psychosocial & $5.25 \pm 2.35$ & $3.19 \pm 2.64$ & $0.000^{*}$ \\
\hline${ }^{\mathrm{d}_{\text {saSPI Intervention }}}$ & $2.84 \pm 2.27$ & $2.98 \pm 2.09$ & 0.744 \\
\hline $\begin{array}{l}\text { Psoriasis Disability } \\
\text { Index (PDI) }\end{array}$ & $12.69 \pm 7.84$ & $6.07 \pm 5.86$ & $<0.001^{*}$ \\
\hline
\end{tabular}

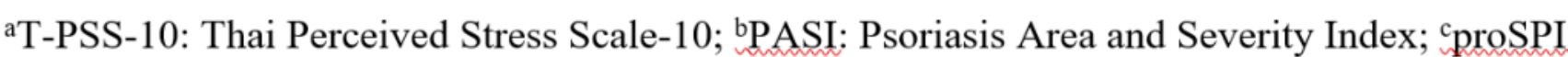

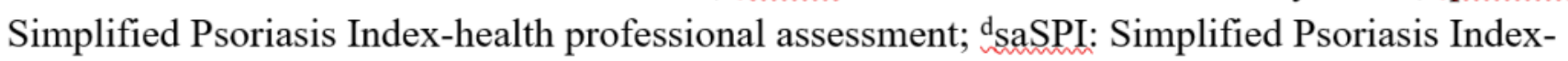
patient self-assessment

\section{Results}

After 150 patients were assessed, $21.33 \%$ of them had depression. Higher scores of stress level, subjective psychosocial impact of psoriasis, selfperceived current severity of psoriasis and more impaired quality of life were significantly associated with depression $(p<0.05)$. Depression severity was positively correlated with the severity of alcohol drinking, perceived stress, quality of life impairment, current severity and psychosocial impact of psoriasis $(p<0.05)$.

\section{Conclusions}

Depression is prevalent among patients with psoriasis. Those with high perceived-stress and high psychosocial impact of psoriasis and more severe disease activity should be evaluated for depression as it can hamper their quality of life.

Table 2: Correlation between severity of depression and other variables

\begin{tabular}{|l|c|c|}
\hline \multicolumn{1}{|c|}{ Variables } & $\begin{array}{c}\text { Coefficient of } \\
\text { Correlation (r) }\end{array}$ & $P$-value \\
\hline $\begin{array}{l}\text { Alcohol Use Disorders Identification } \\
\text { Test (AUDIT) }\end{array}$ & 0.227 & $0.005^{*}$ \\
\hline Psoriasis Disability Index (PDI) & 0.523 & $<0.001^{*}$ \\
\hline T-PSS-10 & 0.336 & $<0.001^{*}$ \\
\hline PASI & 0.161 & 0.093 \\
\hline proSPI Severity & 0.311 & $<0.050$ \\
\hline proSPI Psychosocial & 0.032 & 0.212 \\
\hline proSPI Intervention & 0.103 & $<0.001^{*}$ \\
\hline saSPI Severity & 0.311 & $<0.001^{*}$ \\
\hline saSPI Psychosocial & 0.352 & 0.171 \\
\hline saSPI Intervention & 0.112 & \\
\hline$* P$-value $<0.05$ & & \\
\hline
\end{tabular}

\section{References}

1. Lamb R, Matcham F, Turner M, Rayner L, Simpson A, Hotopf M et al. Screening for anxiety and depression in people with psoriasis: a cross-sectional study in a tertiary referral setting. $\mathrm{Br} \mathrm{J}$ Dermatol. 2017;176(4):1028-34.

2. McDonough E, Ayearst R, Eder L, Chandran V, Rosen CF, Thavaneswaran A et al. Depression and anxiety in psoriatic disease: prevalence and associated factors. J Rheumatol. 2014;41(5):887-96. 